\title{
Remifentanil-induced alterations in neutrophil numbers after surgery
}

\author{
Toshiichro Inagi ${ }^{1}$, Hideko Hoshina ${ }^{2}$, Manzo Suzuki ${ }^{*}$, Miki Wada ${ }^{1}$, Hiroyasu Bito ${ }^{1}$ and Atsuhiro Sakamoto ${ }^{3}$
}

\begin{abstract}
Background: Neutrophils are the first line of defense against invasive microorganisms during and after surgery. There is a possibility that different opioid analgesics used during surgery have different effects on the leucocyte count. We retrospectively analyzed the numbers of leucocytes, neutrophils, and lymphocytes just after surgery in patients who received remifentanil-based anesthesia and those who received fentanyl-based anesthesia. In female patients who underwent modified mastectomy or simple mastectomy with resection of a lymph node(s) or with biopsy of a sentinel lymph node(s) between January 2010 and December $2013(n=83)$, propensity score pairwise matching was performed according to the patient's age and procedure, and forty patients (Remifentanil group and Fentanyl group; $n=20$ each) were analyzed.

Findings: Postoperative numbers of leucocytes and neutrophils were significantly lower in patients who received remifentanil-based anesthesia than in those who received fentanyl-based anesthesia ( $p=0.03, p=0.014$; leucocytes and neutrophils, respectively). The increases in the numbers of leucocytes and neutrophils were significantly lower in the patients in the remifentanil group ( $p=0.009, p=0.0046$; increase in leucocytes and neutrophils, respectively).

Conclusions: In conclusion, remifentanil-based anesthesia attenuates postoperative leucocyte and neutrophil counts. It is unknown whether this phenomenon indicates the possibility of immunosuppression. Further studies are required.
\end{abstract}

Keywords: Neutrophil counts, Remifentanil, Surgery, Anesthesia

\section{Findings}

\section{Introduction}

Neutrophils are the first line of defense against invasive microorganisms during and after surgery. The numbers of leucocytes, neutrophils and lymphocytes may change in response to surgery due to various hormones, cytokines and acute phase reactants [1].

Neutrophils are produced within hematopoietic cords interspersed within venous sinuses of the bone marrow, and the number of circulating neutrophils is maintained by a fine balance among granulopoiesis, bone marrow storage and release, intravascular margination, clearance, apoptosis and destruction [2]. The principal regulator of granulopoiesis is granulocyte colony stimulating factor (G-CSF) [3]. Interleukin (IL)-6 and IL-8 are also known to promote neutrophil production in vitro and in vivo $[4,5]$.

\footnotetext{
* Correspondence: manzo@nms.ac.jp

${ }^{1}$ Department of Anesthesiology, Musashikosugi Hospital Nippon Medical School, 1-396 Kosugi-cho Nakahara-ku, Kawaski, Kanagawa 211-8533, Japan Full list of author information is available at the end of the article
}

The production of immune mediators is associated with the extent of surgical trauma, and is also influenced by the anesthesia technique [6,7]. Remifentanil is a newly developed analgesic that possesses extremely rapid clearance. An in vitro study suggested that remifentanil decreased expression of inflammatory cytokines such as TNF- $\alpha$, IL- 6 and IL- 8 induced by lipopolysaccharide (LPS) in human neutrophils [8].

These results suggest that the different opioid analgesics used during surgery may have different effects on the leucocyte count. We retrospectively analyzed the numbers of leucocytes, neutrophils, and lymphocytes just after surgery in patients who received remifentanilbased anesthesia and those who received fentanyl-based anesthesia.

\section{Methods}

The records of female patients who underwent modified mastectomy or simple mastectomy with resection of a lymph node(s) or with biopsy of a sentinel lymph

\section{Springer}


node(s) between January 2010 and December 2013 were retrospectively investigated. This study was approved by the ethics committee of Musashikosugi Hospital Nippon Medical School. Exclusion criteria included patients who received chemotherapy before surgery, patients who presented with a febrile symptom before the surgery (C-related protein $>0.3$ ), and patients over the age of 75 years. Patients whose surgery lasted less than $80 \mathrm{~min}$ or longer than 181 min were excluded.

The following data were collected and compared between the patients who received remifentanil-based anesthesia and those who received fentanyl-based anesthesia: patients' age, height, weight, duration of surgery, amount of infusion, blood loss, and total amount of opioid analgesic (fentanyl, remifentanil, or both) administered during surgery. The systolic blood pressure before the surgery, highest systolic blood pressure during surgery, systolic blood pressure just after the surgery, and heart rates at the respective systolic blood pressure were recorded. The mean infusion rate of remifentanil $(\mu \mathrm{g} / \mathrm{kg} / \mathrm{h})$ was calculated by dividing the total amount of remifentanil administered by the patient's weight and the duration of the surgery. Preoperative and postoperative counts of leucocytes, neutrophils and lymphocytes were recorded. The increases in numbers of leucocytes, neutrophils, and lymphocytes were calculated by subtracting the preoperative value from the respective postoperative value. Patients who received additional pain medicine (e.g., NSAIDs) were noted.

\section{Statistical analysis}

To reduce the selection bias on the number of immune cells, we performed a propensity score pairwise matching of patients maintained with remifentanil with patients maintained with fentanyl, using patient's age and procedure. As the propensity score-matched analysis was selected to reduce preoperative confounding factors, the propensity score was constructed with the aid of a logistic regression model using preoperative variables. Patients maintained with remifentanil whose propensity

Table 1 Characteristics of the patients who received remifentanil-based anesthesia or fentanyl-based anesthesia

\begin{tabular}{|c|c|c|c|c|}
\hline & & Remifentanil $(n=47)$ & Fentanyl $(n=36)$ & $P$ value \\
\hline \multicolumn{2}{|l|}{$\overline{\text { Age }(y)}$} & $57 \pm 11$ & $51 \pm 10$ & 0.025 \\
\hline \multicolumn{2}{|l|}{ Height $(\mathrm{cm})^{\mathrm{a}}$} & $156 \pm 6$ & $154 \pm 7$ & 0.21 \\
\hline \multicolumn{2}{|l|}{ Weight $(\mathrm{kg})^{\mathrm{a}}$} & $55 \pm 8$ & $55 \pm 10$ & 0.98 \\
\hline \multicolumn{2}{|c|}{ Mastectomy: partial mastectomy } & $26: 21$ & $13: 23$ & 0.39 \\
\hline \multicolumn{2}{|l|}{ SLNB or RLN ${ }^{b}$} & 23:24 & 21:15 & 0.08 \\
\hline \multicolumn{2}{|l|}{ Received flubiprofen } & 19 & 15 & 0.9 \\
\hline \multicolumn{2}{|c|}{ Total amount of remifentanil $(\mu \mathrm{g} / \mathrm{kg})^{a}$} & $23.5 \pm 11.4$ & $0 \pm 0$ & $<0.0001$ \\
\hline \multicolumn{2}{|c|}{ Total amount of fentanyl $(\mu \mathrm{g} / \mathrm{kg})^{\mathrm{a}}$} & $2.2 \pm 2.8$ & $5.5 \pm 2.5$ & $<0.0001$ \\
\hline \multicolumn{2}{|c|}{ Duration of surgery $(\mathrm{min})^{\mathrm{a}}$} & $111 \pm 33$ & $122 \pm 30$ & 0.1 \\
\hline \multicolumn{2}{|l|}{ Mac-hour(h) } & $2.1 \pm 0.5$ & $2.6 \pm 0.7$ & 0.01 \\
\hline \multicolumn{2}{|c|}{ Total amount of ephedrine (mg) } & $3 \pm 3.8$ & $1.3 \pm 2.2$ & 0.06 \\
\hline \multicolumn{2}{|c|}{ Number of Patients received ephedrine (n) } & 21 & 12 & 0.36 \\
\hline \multirow[t]{4}{*}{ Blood pressure $(\mathrm{mmHg})$} & Preope & $144 \pm 24$ & $139 \pm 25$ & 0.36 \\
\hline & Highest & $122 \pm 17$ & $131 \pm 18$ & 0.019 \\
\hline & Lowest & $82 \pm 7$ & $88 \pm 10$ & 0.0024 \\
\hline & Postope & $133 \pm 17$ & $133 \pm 24$ & 0.95 \\
\hline \multirow[t]{4}{*}{ Heart rate (b/min) } & Preope & $73 \pm 13$ & $70 \pm 13$ & 0.35 \\
\hline & At highest BP & $61 \pm 9$ & $64 \pm 10$ & 0.14 \\
\hline & At lowest BP & $55 \pm 7$ & $56 \pm 7$ & 0.42 \\
\hline & Postope & $74 \pm 12$ & $72 \pm 14$ & 0.43 \\
\hline \multicolumn{2}{|c|}{ Total amount of infusion $(\mathrm{ml})^{\mathrm{a}}$} & $1250 \pm 385$ & $1325 \pm 390$ & 0.4 \\
\hline \multicolumn{2}{|c|}{ Preoperative leucocyte count $(/ \mu l)^{a}$} & $5726 \pm 1656$ & $5536 \pm 1405$ & 0.63 \\
\hline \multicolumn{2}{|c|}{ Preoperative neutrophil count $(/ \mu l)^{\mathrm{a}}$} & $3270 \pm 1230$ & $3210 \pm 1215$ & 0.83 \\
\hline \multicolumn{2}{|c|}{ Preoperative lymphocyte count $(/ \mu l)^{a}$} & $1910 \pm 690$ & $1820 \pm 545$ & 0.51 \\
\hline \multicolumn{2}{|c|}{ Preoperative monocyte count $(/ \mu l)^{a}$} & $260 \pm 80$ & $270 \pm 110$ & 0.64 \\
\hline
\end{tabular}

${ }^{\mathrm{a} M e a n} \pm \mathrm{SD}$

${ }^{\mathrm{b}} S L N B$ sentinel lymph node biopsy, $R L N$ resection of lymph node 
scores deviated by $>0.02$ from those of patients maintained with fentanyl, were considered unmatched.

Results are shown as mean \pm standard deviation. The significance of differences in categorical values and numerical values were analyzed using chi-squared test and unpaired $t$-test, respectively. The correlation between numerical parameters was analyzed using linear regression analysis. Significance was set at $p<0.05$.

\section{Results}

Eighty-three patients underwent modified mastectomy or simple mastectomy with resection of a lymph node or with biopsy of a sentinel lymph node met the study criteria. Of these, 36 patients received fentanyl-based anesthesia and 47 patients received remifentanil-based anesthesia. All patients received propofol and rocuronium, and trachea was intubated. Anesthesia was maintained by sevoflurane, propofol had not been used for maintenance. Blood samples had been obtained within
$2 \mathrm{~h}$ after emergence from anesthesia in all patients. The characteristics of the patients who received remifentanilbased anesthesia or fentanyl-based anesthesia are summarized in Table 1. After propensity matching, we obtained 40 patients for analysis, which included 20 patients in the remifentanil group who received remifentanil-based anesthesia, and 20 patients in the fentanyl group who received fentanyl-based anesthesia. No patients in neither fentanyl nor remifentanil group developed problem in wound healing process such as surgical site infection. No patients received catecholamine as inotropes, only ephedrine had been used. As shown in Table 2, there is no difference in number of patients who received ephedrine and in amount of ephedrine. Postoperative numbers of leucocytes and neutrophils were significantly lower in remifentanil group than fentanyl group (leucocytes, $7521 \pm 2492$ vs. $5873 \pm 2123, p=0.03$; neutrophils, $5098 \pm 1964$ vs. $3499 \pm 1970, p=0.014$ ) (Fig. 1). There was no significant difference in the number of lymphocytes between the two

Table 2 Characteristics of the patients who received remifentanil-based anesthesia or fentanyl-based anesthesia after propensity matching

\begin{tabular}{|c|c|c|c|c|}
\hline \multirow{2}{*}{\multicolumn{2}{|c|}{$\overline{\text { Age (y) }}$}} & Remifentanil group $(n=20)$ & Fentanyl group $(n=20)$ & $P$ value \\
\hline & & $54 \pm 11$ & $52 \pm 12$ & 0.68 \\
\hline \multicolumn{2}{|l|}{ Height $(\mathrm{cm})^{\mathrm{a}}$} & $157 \pm 7.5$ & $152 \pm 5$ & 0.38 \\
\hline \multicolumn{2}{|l|}{ Weight $(\mathrm{kg})^{\mathrm{a}}$} & $54 \pm 9$ & $56 \pm 11$ & 0.58 \\
\hline \multicolumn{2}{|c|}{ Mastectomy: partial mastectomy } & $5: 15$ & $6: 14$ & 0.72 \\
\hline \multicolumn{2}{|l|}{ SLNB or RLN ${ }^{b}$} & 19:1 & $18: 2$ & 0.54 \\
\hline \multicolumn{2}{|l|}{ Received flubiprofen } & 19 & 15 & 0.9 \\
\hline \multicolumn{2}{|c|}{ Total amount of remifentanil $(\mu \mathrm{g} / \mathrm{kg})^{a}$} & $20.1 \pm 9.4$ & $0 \pm 0$ & $<0.0001$ \\
\hline \multicolumn{2}{|c|}{ Total amount of fentanyl $(\mu \mathrm{g} / \mathrm{kg})^{\mathrm{a}}$} & $1.9 \pm 1.9$ & $5.4 \pm 2.1$ & $<0.0001$ \\
\hline \multicolumn{2}{|c|}{ Duration of surgery $(\mathrm{min})^{\mathrm{a}}$} & $94 \pm 23$ & $103 \pm 20$ & 0.14 \\
\hline \multicolumn{2}{|l|}{ MAC-hour(h) } & $2.2 \pm 0.5$ & $2.7 \pm 0.7$ & 0.01 \\
\hline \multicolumn{2}{|c|}{ Total amount of ephedrine (mg) } & $3 \pm 3.5$ & $1.2 \pm 2.1$ & 0.06 \\
\hline \multicolumn{2}{|c|}{ Number of Patients received ephedrine (n) } & 9 & 5 & 0.32 \\
\hline \multirow[t]{4}{*}{ Blood pressure $(\mathrm{mmHg})$} & Preope & $137 \pm 20$ & $139 \pm 24$ & 0.8 \\
\hline & Highest & $116 \pm 15$ & $135 \pm 17$ & 0.0016 \\
\hline & Lowest & $80 \pm 7$ & $87 \pm 10$ & 0.02 \\
\hline & Postope & $130 \pm 16$ & $134 \pm 25$ & 0.64 \\
\hline \multirow[t]{4}{*}{ Heart rate (b/min) } & Preope & $76 \pm 16$ & $69 \pm 12$ & 0.10 \\
\hline & At highest BP & $63 \pm 10$ & $65 \pm 11$ & 0.22 \\
\hline & At lowest BP & $55 \pm 7.5$ & $56 \pm 7.5$ & 0.8 \\
\hline & Postope & $74 \pm 15$ & $72 \pm 10$ & 0.48 \\
\hline \multicolumn{2}{|c|}{ Total amount of infusion (ml) ${ }^{a}$} & $1235 \pm 290$ & $1360 \pm 460$ & 0.3 \\
\hline \multicolumn{2}{|c|}{ Preoperative leucocyte count $(/ \mu l)^{a}$} & $5460 \pm 1460$ & $5622 \pm 1587$ & 0.74 \\
\hline \multicolumn{2}{|c|}{ Preoperative neutrophil count $(/ \mu l)^{a}$} & $3169 \pm 1192$ & $3274 \pm 1264$ & 0.78 \\
\hline \multicolumn{2}{|c|}{ Preoperative lymphocyte count $(/ \mu l)^{a}$} & $1845 \pm 663$ & $1857 \pm 619$ & 0.95 \\
\hline \multicolumn{2}{|c|}{ Preoperative monocyte count $(/ \mu l)^{\mathrm{a}}$} & $265 \pm 81$ & $267 \pm 106$ & 0.94 \\
\hline
\end{tabular}

a Mean \pm SD

${ }^{\mathrm{b}} S L N B$ sentinel lymph node biopsy, $R L N$ resection of lymph node 


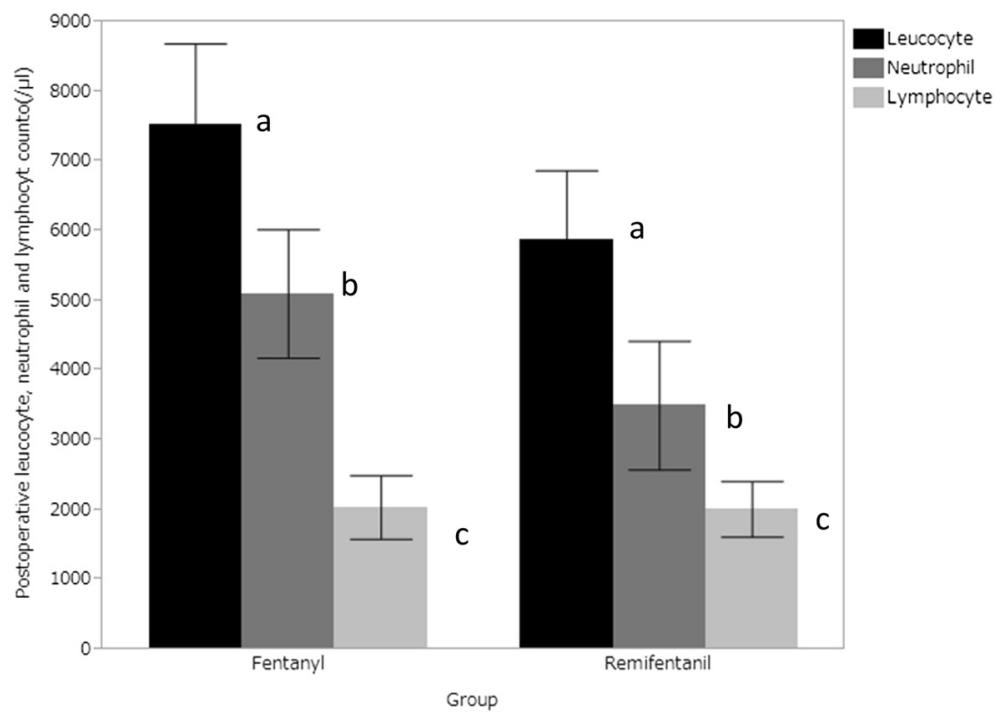

Fig. 1 Comparison of the postoperative numbers of leucocytes (black bar), neutrophils (gray bar) and lymphocytes (white bar) between the patients who received fentanyl-based anesthesia $(n=20)$ and those who received remifentanil-based anesthesia $(n=20)$. The leucocyte count (a: $p=0.03$ ) and neutrophil count ( $b: p=0.014)$ were significantly lower in patients who received remifentanil-based anesthesia than in those who received fentanyl-based anesthesia. Error bars represent the standard deviation. There was no significant difference in the number of lymphocytes between the two groups (c: $p=0.92)$

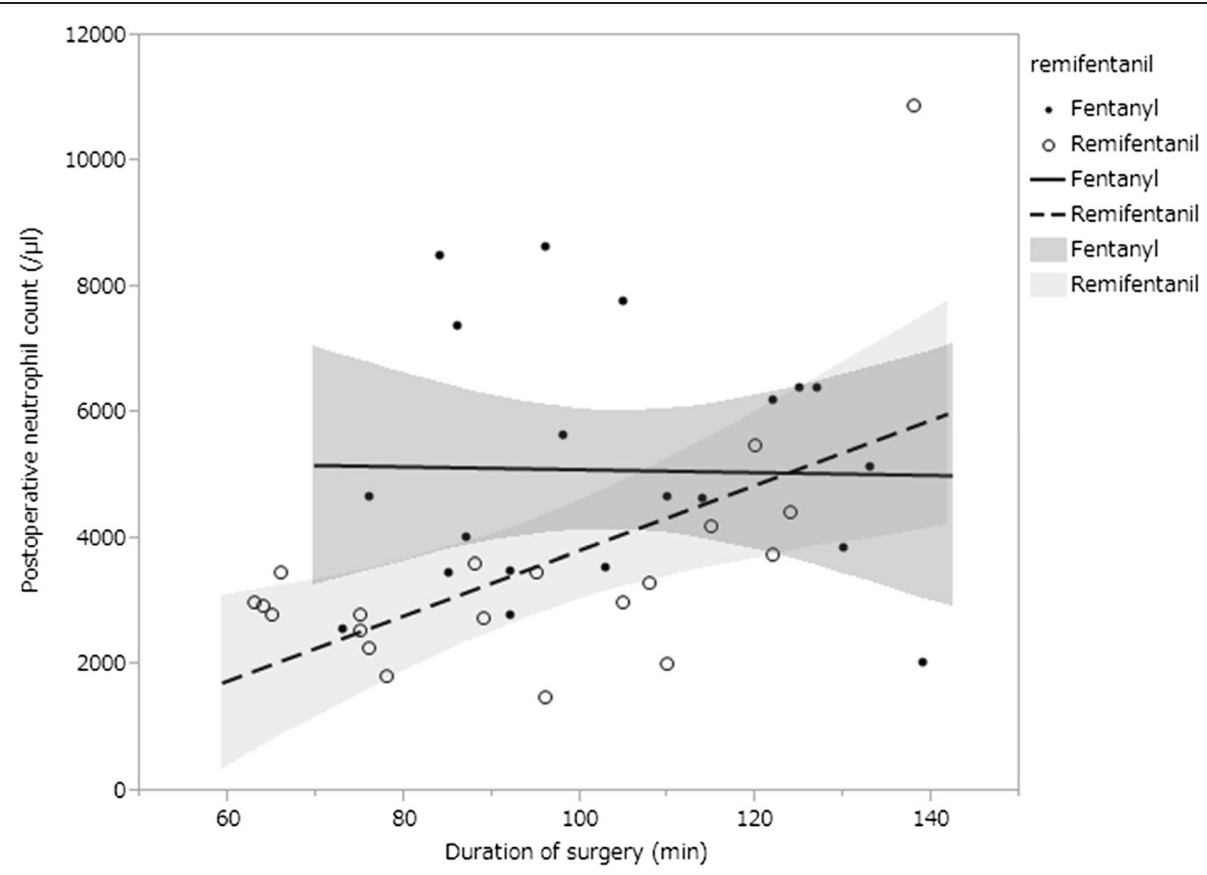

Fig. 2 Correlations between the postoperative neutrophil count and duration of surgery in fentanyl group (thick line and dark shadow), and between the postoperative neutrophil count and duration of surgery in remifentanil group (dotted line and light shadow). The dark shadow represents the $95 \%$ confidence interval $(\mathrm{Cl})$ in fentanyl group and the light shadow represents the $95 \% \mathrm{Cl}$ in remifentanil group. Neutrophil count $=5335-2.2^{*}$ duration of surgery $(\mathrm{min}) . R^{2}=0.00, P=0.92$ (Fentanyl group). Neutrophil count $=-1349+51.8^{*}$ duration of surgery (min). $R^{2}=0.36, P=0.0044$ (Remifentanil group) 
groups (Fig. 1). Among the 20 patients in the remifentanil group, 13 patients received additional fentanyl. In the remifentanil group, there were no differences in the numbers of leucocytes, neutrophils and lymphocytes between the patients who received additional fentanyl and those who did not. The postoperative number of of neutrophils was correlated with the duration of surgery only in the remifentanil group ( $\mathrm{R} 2=0.36, p=0.004)$ (Fig. 2).

\section{Discussion}

In the present study, we observed that postoperative counts of leucocytes and neutrophils were significantly lower in the patients who received remifentanil-based anesthesia than in those who received fentanyl-based anesthesia. Because the number of lymphocytes and increase in the number of lymphocytes from the preoperative value did not differ between those who received remifentanil-based anesthesia and those who received fentanyl-based anesthesia, the difference in the number of leucocytes may be mainly due to the kinetics of neutrophils.

One explanation for why the neutrophil count was higher in the fentanyl group is an increase in release of neutrophils from bone marrow by inflammatory cytokines in the fentanyl group. Terashima et al. [9] demonstrated that administration of IL-8 rapidly increased the numbers of granulocytes and polymorphonuclear leukocytes without affecting transit time through both the mitotic and post-mitotic pools of bone marrow. In addition to G-CSF, IL-6 and IL-3 stimulate granulopoiesis in vivo [10]. Remifentanil attenuates expression of pro-inflammatory cytokines including TNF- $\alpha$, IL- 6 , and IL-8 in vitro [8]. Another in vitro study demonstrated decreased neutrophil transmigration through the endothelial cell monolayer by co-incubation with remifentanil [11], and this suggests that a smaller number of neutrophils migrated to damaged tissue from microvessels in the remifentanil group than in the fentanyl group. The possibility that a low number of neutrophils reached damaged tissue in the remifentanil group suggests that the amount of cytokines released from neutrophils recruited in the damaged site may have been lower in the remifentanil group than in the fentanyl group. Among patients who underwent cardiac surgery, patients who received fentanyl-based anesthesia had a higher level of stress hormone and higher levels of proinflammatory cytokines compared with patients who received remifentanilbased anesthesia [12]. In the present study, the numbers of leucocytes and neutrophils were both correlated with the duration of surgery among the patients in the remifentanil group. Long duration of surgery may be related to surgical stress. In the present study, one possible reason for the difference in the number of neutrophils between the remifentanil group and fentanyl group is a difference in production of neutrophils in bone marrow introduced by differences in surgical stress and inflammatory cytokines.

There have only been a few studies on the relationship between remifentanil and immunosuppression. Studies in vitro demonstrated that high-dose remifentanil suppresses the release of cytokines from neutrophils and monocytes that had been stimulated by LPS [8]. Very lowdose remifentanil did not suppress NK-cell activity in healthy human volunteers [13]. A study in rats demonstrated that continuous infusion of remifentanil inhibited the proliferation of splenocytes induced by concanavalin A [14]. Although the reductions in the elevation of leucocytes and neutrophils in the remifentanil group may have been related to reduction in the proliferation of neutrophils, whether suppression of increases in the numbers of leucocytes and neutrophils directly causes immunosuppression is unknown. General anesthetic agents induce different inflammatory responses [6, 7]. Sevoflurane has a smaller effect on the neutrophil count than desflurane [7]. The Mac-hour of sevoflurane in the remifentanil group was significantly lower than that in the fentanyl group, although the difference was very small. We believe that the effect of sevoflurane on the results may have been small.

In conclusion, remifentanil-based anesthesia attenuated postoperative leucocyte and neutrophil counts. It is unknown whether this phenomenon indicates the possibility of immunosuppression. Further studies are required.

\section{Consent}

Written informed consent was obtained from the patient for publication of case report and any accompanying images. A copy of written consent is available for review by the editor-in-chief of this journal.

\section{Competing interests \\ The authors declare that they have no competing interests.}

Authors' contributions

MS participated in designing the study and writing the first draft of the manuscript. HH carried out blood sampling. MW, YN, and HT obtained perioperative data from medical records. HB participated in designing the study and helped to draft the manuscript. All authors have read and approved the final manuscript

\section{Acknowledgements}

We thank N.A.I co. for reviewing this manuscript.

This manuscript has been carefully reviewed by an experienced editor whose first language is English and who specializes in editing papers written by scientists whose native language is not English.

\section{Author details}

${ }^{1}$ Department of Anesthesiology, Musashikosugi Hospital Nippon Medical School, 1-396 Kosugi-cho Nakahara-ku, Kawaski, Kanagawa 211-8533, Japan. ${ }^{2}$ Department of Breast Surgery, Musashikosugi Hospital Nippon Medical School, Kanagawa, Japan. ${ }^{3}$ Department of Aneasthesiology, Nippon Medical School, 1-1-5 Sendagi Bunkyo-ku, Tokyo 113-8102, Japan.

Received: 28 October 2015 Accepted: 22 February 2016

Published online: 02 April 2016 


\section{References}

1. Ogawa K, Hirai M, Katsube T, Murayama M, Hamaguchi K, Shimakawa T, Naritake Y, Hosokawa T, Kajiwara T. Suppression of cellular immunity by surgical stress. Surgery. 2000;127:329-36.

2. Iwase M, Kondo G, Watanabe H, Takaoaka S, Uchida M, Ohashi M, Nagumo M. Regulation of Fas-related apoptosis in neutrophils after surgery-induced acute inflammation. J Surg Res. 2006;134:114-23.

3. Walker F, Zhang HH, Mathews V, Weinstock J, Nice EC, Ernst M, Rose-John S, Burgess AW. IL6/SIL6R complex contributes to emergency granulopoietic responses in G-CSF-and GM-CSF-deficient mice. Blood. 2008;111:3978-85.

4. Summers C, Rankin SM, Condliffe AM, Singh N, Peters AM, Chilvers ER. Neutrophil kinetics in health and disease. Trends Immunol. 2010;31:318-24.

5. Romani L, Mencacci A, Cenci E, Spaccapelo R, Toniatti C, Puccetti P, Biston F, Poli V. Impaired neutrophil response and $\mathrm{CD}^{+} \mathrm{T}$ helper cell development in interleukine 6-deficient mice infected with candida albicans. J Exp Med. 1996;183:1345-55

6. Kim WH, Jin HS, Ko JS, Hahm TS, Lee SM, Cho HS, Kim MH. The effect of anesthetic techniques on neutrophil-to-lymphocyte ration after laparoscopy-assisted vaginal hysterectomy. Acta Anaesthesil Taiwan. 2011:49:83-7.

7. Cocelli LP, Ugur MG, Karadasli H. Comparison of effects of low-flow sevoflurane and desflurane on neutrophil and T-cell populations. Curr Ther Res. 2012;73:41-51

8. Hyejin J, Mei L, Seongheon L, Cheolwon J, Seokjai K, Hongbeom B, Minsun K, Sungsu C, Sanghyun K. Remifentanil attenuates human neutrophils activation induced by lipopolysaccharide. Immunopharmacol Immunotoxicol. 2013;35:264-71.

9. Terashima T, English D, Hogg JC, van Eeden SF. Release of polymorphonuclear leukocytes from the bone marrow by interleukin-8. Blood. 1998;92:1062-9.

10. Pojda Z, Tsuboi A. In vivo effects of human recombinant interleukin 6 on hemopoietic stem and progenitor cells and circulating blood cells in normal mice. Exp Hematol. 1990:18:1034-7.

11. Hofbauer R, Frass M, Gmeiner B, Sandor N, Schumann R, Wagner O, Kaye AD. Effects of remifentanil on neutrophil adhesion, transmigration, and intercellular adhesion molecule expression. Acta Anaesthesiol Scand. 2000:44:1232-7.

12. Winterhalter M, Brandl K, Rahe-Meyer N, Osthaus A, Hecker H, Hagl C, Adams HA, Piepenbrock $\mathrm{S}$. Endocrine stress response and inflammatory activation during CABG surgery. A randomized trial comparing remifentanil infusion to intermittent fentanyl. Eur J Anaesthesiol. 2008;25:326-35.

13. Cronin AJ, Aucutt-Walter NM, Budinetz T, Bonafide CP, DiVittore NA, Gordin $\checkmark$, Schuler HG, Bonneau RH. Low-dose remifentanil infusion does not impair natural killer cell function in healthy volunteers. Br J Anaesth. 2003:91:805-9.

14. Sacerdote P, Gaspani L, Rossoni G, Panerai AE, Bianchi M. Effect of the opioid remifentanil on cellular immune response in the rat. Int Immunopharmacol. 2001;1:713-9.

\section{Submit your manuscript to a SpringerOpen ${ }^{\circ}$ journal and benefit from:}

- Convenient online submission

- Rigorous peer review

- Immediate publication on acceptance

- Open access: articles freely available online

- High visibility within the field

- Retaining the copyright to your article 